\title{
Digital Phase Modulation
}




\section{Applications of Communications Theory Series Editor: R. W. Lucky, $A T$ \& $T$ Bell Laboratories}

COMPUTER NETWORK ARCHITECTURES AND PROTOCOLS

Edited by Paul E. Green, Jr.

DATA TRANSPORTATION AND PROTECTION

John E. Hershey and R. K. Rao Yarlagadda

DEEP SPACE TELECOMMUNICATIONS SYSTEMS ENGINEERING

Edited by Joseph H. Yuen

DIGITAL PHASE MODULATION

John B. Anderson, Tor Aulin, and Carl-Erik Sundberg

ERROR-CORRECTION CODING FOR DIGITAL COMMUNICATIONS

George C. Clark, Jr., and J. Bibb Cain

FIBER OPTICS: Technology and Applications

Stewart D. Personick

FUNDAMENTALS OF DIGITAL SWITCHING

Edited by John C. McDonald

INTRODUCTION TO COMMUNICATION SCIENCE AND SYSTEMS

John R. Pierce and Edward C. Posner

MODELING AND ANALYSIS OF COMPUTER

COMMUNICATIONS NETWORKS

Jeremiah F. Hayes

MODERN TELECOMMUNICATION

E. Bryan Carne

OPTICAL FIBER TRANSMISSION SYSTEMS

Stewart D. Personick

PRACTICAL COMPUTER DATA COMMUNICATIONS

William J. Barksdale

TELECOMMUNICATIONS SWITCHING

J. Gordon Pearce

A Continuation Order Plan is available for this series. A continuation order will bring delivery of each new volume immediately upon publication. Volumes are billed only upon actual shipment.

For further information please contact the publisher. 


\section{Digital Phase Modulation}

\section{John B. Anderson}

Rensselaer Polytechnic Institute

Troy, New York

\section{Tor Aulin}

Chalmers University of Technology

Göteborg, Sweden

and

\section{Carl-Erik Sundberg}

AT \& T Bell Laboratories

Holmdel, New Jersey

Springer Science+Business Media, LLC 


\section{Library of Congress Cataloging in Publication Data}

Anderson, John B., 1945-

Digital phase modulation.

(Applications of communications theory)

Includes bibliographical references and index.

1. Digital modulation. 2. Phase modulation. 3. Modulation theory. I. Aulin, Tor. II. Sundberg, Carl-Erik. III. Title. IV. Series.

1098765432

ISBN 978-1-4899-2033-1 ISBN 978-1-4899-2031-7 (eBook)

DOI 10.1007/978-1-4899-2031-7

(C) 1986 Springer Science+Business Media New York

Originally published by Plenum Press, New York in 1986

Softcover reprint of the hardcover 1st edition 1986

All rights reserved

No part of this book may be reproduced, stored in a retrieval system, or transmitted in any form or by any means, electronic, mechanical, photocopying, microfilming, recording, or otherwise, without written permission from the Publisher 


\section{Preface}

The last ten years have seen a great flowering of the theory of digital data modulation. This book is a treatise on digital modulation theory, with an emphasis on these more recent innovations. It has its origins in a collaboration among the authors that began in 1977. At that time it seemed odd to us that the subjects of error-correcting codes and data modulation were so separated; it seemed also that not enough understanding underlay the mostly ad hoc approaches to data transmission. A great many others were intrigued, too, and the result was a large body of new work that makes up most of this book. Now the older disciplines of detection theory and coding theory have been generalized and applied to the point where it is hard to tell where these end and the theories of signal design and modulation begin.

Despite our emphasis on the events of the last ten years, we have included all the traditional topics of digital phase modulation. Signal space concepts are developed, as are simple phase-shift-keyed and pulse-shaped modulations; receiver structures are discussed, from the simple linear receiver to the Viterbi algorithm; the effects of channel filtering and of hardlimiting are described. The volume thus serves well as a pedagogical book for research engineers in industry and second-year graduate students in communications engineering.

The production of a manageable book required that many topics be left out. The major constraint in the book is that it is strictly limited to phase modulation. Schemes in which data symbols cause changes in signal amplitude are omitted. Much other exciting new work had to be left out: Channel equalization, new hardware, new error-correcting codes, and multiple-access problems are a few of these subjects. Some readers may seek a more thorough grounding in the mathematical basis of communications theory, since the book assumes a basic knowledge of vector spaces, probability, and random processes. In addition, the rapid treatment of detection theory, information theory, and phase-lock loops may not be enough for some readers. 
The book is organized as follows. An introduction expounds the reasons, both technical and commercial, why digital transmission has become so important. Communications theory is the subject of Chapter 2; detection and signal space theory are introduced, along with the error probability and spectrum of a modulation and some simple receivers for it. The older, simpler modulation schemes like phase-shift keying and their real-world behavior provide illustrations of these theories. Chapters 3 and 4 then develop in detail the energy and bandwidth performance of a very general class of modulations called continuous-phase modulations or CPM. Chapter 5 looks at the simultaneous energy and bandwidth performance of these modulations, from the standpoint of both particular schemes and the Shannon theory of modulation. Chapter 6 deals with CPM transmitter structures, while Chapters 7 and 8 discuss more sophisticated receivers than those in Chapter 2. Synchronization and performance with partial phase knowledge form the subject matter of Chapters 9 and 10. Chapter 11 explores the effect of modulations on error-correcting codes. A half-year course in digital modulation could focus on Chapters 1-5 and 7, with other topics chosen to suit the instructor's taste. A shorter, more theoretical course could feature Chapters 2-4.

In writing this book, we have attempted to give some idea of the historical development of the subject, but we have not performed a rigorous literature search and we apologize to the many contributors to the field who have not been referenced by name. We are deeply indebted to our colleagues and co-workers at McMaster University in Canada, the University of Lund and Chalmers Technical University in Sweden, and Rensselaer Polytechnic Institute in the U.S.A., and to our industrial co-workers at INTELSAT, AT \& T-Bell Laboratories (U.S.A.), the General Electric (U.S.A.) Corporate Research and Development Center, Codex Corporation (U.S.A.), Ericsson Radio Systems (Sweden), and the European Space Agency. Of this large body of co-workers we must give special mention to Rudi de Buda, Nils Rydbeck, Arne Svensson, and Göran Lindell. It is a special pleasure to acknowledge the typing services of Doris Glöck, Constance Brough, Inga-Britt Holmdahl, Annette Laiacona, and Sandi-jo Rumsey and the patience and guidance of our editor, $L$. S. Marchand. Certain government agencies were instrumental in the support of the new research in the book; these were the Swedish Board of Technical Development (STU) and the Natural Sciences and Engineering Research Council of Canada.

J. B. Anderson Troy, New York

T. Aulin Göteborg, Sweden

C.-E. Sundberg Holmdel, New Jersey 


\section{Contents}

Chapter 1. Introduction $\ldots \ldots \ldots \ldots \ldots \ldots \ldots \ldots \ldots \ldots \ldots \ldots \ldots \ldots \ldots$

1.1. Why Digital Transmission? .............................. 1

1.2. Some Basic Digital Channels $\ldots \ldots \ldots \ldots \ldots \ldots \ldots \ldots \ldots \ldots \ldots \ldots \ldots \ldots, 4$

1.3. Some Digital Channel Concepts $\ldots \ldots \ldots \ldots \ldots \ldots \ldots \ldots \ldots \ldots \ldots \ldots \ldots, 6$

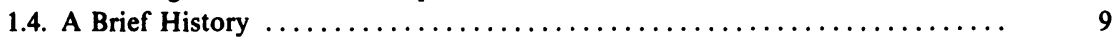

1.5. Organization of the Book $\ldots \ldots \ldots \ldots \ldots \ldots \ldots \ldots \ldots \ldots \ldots \ldots \ldots \ldots, 12$

References $\ldots \ldots \ldots \ldots \ldots \ldots \ldots \ldots \ldots \ldots \ldots \ldots \ldots \ldots \ldots \ldots \ldots \ldots \ldots, 14$

Chapter 2. Signal Analysis and an Overview of Modulation Methods 15

2.1. Receiver Principles for Gaussian Noise ......................... 15

2.1.1. The Maximum Likelihood Receiver ...................... 15

2.1.2. Probability of Error $\ldots \ldots \ldots \ldots \ldots \ldots \ldots \ldots \ldots \ldots \ldots \ldots \ldots, 20$

2.2. Phase Modulated Signals $\ldots \ldots \ldots \ldots \ldots \ldots \ldots \ldots \ldots \ldots \ldots \ldots \ldots \ldots, 22$

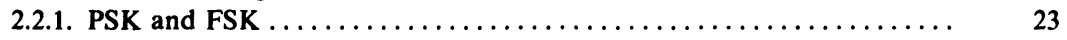

2.2.2. A Notation for Continuous Phase Modulations ............... 25

2.2.3. Euclidean Distance for Phase Modulations .................. 26

2.3. Examples of Error Probability Calculations ..................... 28

2.4. Baseband Receiver and Transmitter Models..................... 31

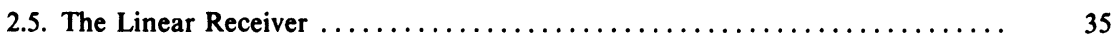

2.5.1. Intersymbol Interference and the Receiver Eye ................. 36

2.5.2. Channel Filter Effects on MSK and PSK .................. 38

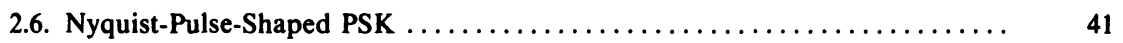

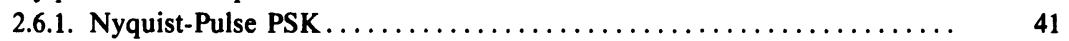

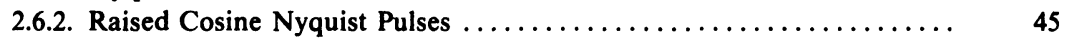

2.7. A Summary of Modulation Methods ........................ 47

2.7.1. Quadrature Modulation Schemes ...................... 48

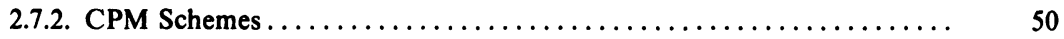

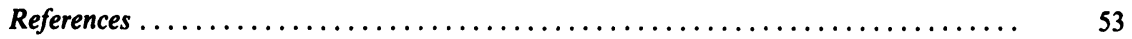

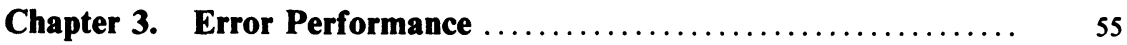

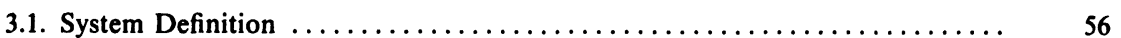


3.2. Bounds on the Minimum Euclidean Distance $\ldots \ldots \ldots \ldots \ldots \ldots \ldots \ldots \ldots .59$

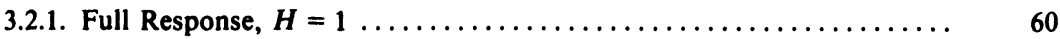

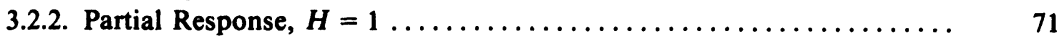

3.2.3. Full Response, Multi-h .......................... 79

3.2.4. Partial Response, Multi-h ......................... 87

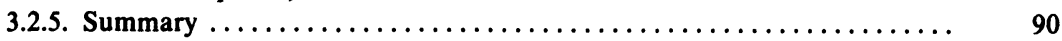

3.3. The Minimum Euclidean Distance ......................... 90

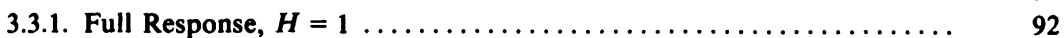

3.3.2. Partial Response, $H=1 \ldots \ldots \ldots \ldots \ldots \ldots \ldots \ldots \ldots \ldots \ldots . \ldots \ldots$

3.3.3. Full Response, Multi-h .......................... 105

3.3.4. Partial Response, Multi-h ........................ 106

3.3.5. Summary ...................................... 109

3.4. Systems with a Trellis Structure $\ldots \ldots \ldots \ldots \ldots \ldots \ldots \ldots \ldots \ldots \ldots \ldots \ldots, 111$

3.4.1. A State Description for Modulators .................... 111

3.4.2. Transfer Function Symbol Error Probability Bounds............ 114

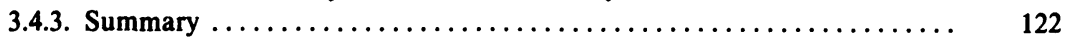

3.5. Effects of Filtering and Limiting .......................... 123

3.5.1. The Euclidean Distance for Mismatched Signal Sets............. 123

3.5.2. Effects of Filtering $\ldots \ldots \ldots \ldots \ldots \ldots \ldots \ldots \ldots \ldots \ldots \ldots \ldots \ldots, 126$

3.5.3. Effects of Filtering and Hard Limitation .................. 136

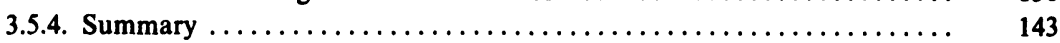

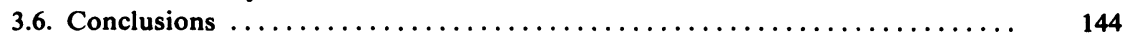

References .............................................. 144

Chapter 4. Spectral Performance ....................... 147

4.1. Review of Spectral Calculation Methods ..................... 147

4.2. A Simple, Fast, and Reliable General Numerical Method .............. 149

4.2.1. Derivation of the Method ........................... 149

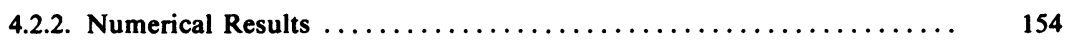

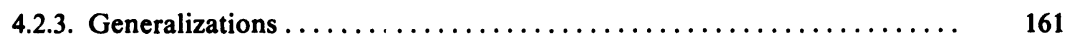

4.2.4. Summary of the Autocorrelation Function Method ............. 163

4.3. Closed Form Expressions for Some Simple Schemes ................. 166

4.4. Asymptotic and Related Behavior of Spectra ..................... 168

4.5. Effects of Filtering and Amplitude Limitation .................... 173

References .......................................... 179

\section{Chapter 5. Energy-Bandwidth Comparisons and Shannon Theory for} Phase Modulation ............................. 181

5.1. Energy and Bandwidth Comparison of Concrete Modulation Systems ...... 182

5.2. Basic Ideas of the Shannon Theory ........................ 186

5.2.1. The Shannon Model of Information Transmission ............ 187

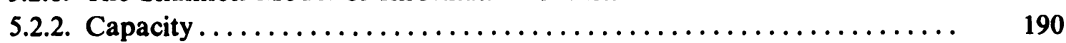

5.2.3. Bandwidth and Dimensionality ....................... 192

5.3. AWGN Channel Capacity and the Phase Modulation Channel ........... 194

5.3.1. AWGN Channel Capacity ............................. 194

5.3.2. A Phase Modulation Channel Model ..................... 195 
5.4. The Cutoff Rate for Phase Modulation Channels $\ldots \ldots \ldots \ldots \ldots \ldots \ldots \ldots \ldots$

5.5. Numerical Results for $\boldsymbol{R}_{\mathbf{0}} \ldots \ldots \ldots \ldots \ldots \ldots \ldots \ldots \ldots \ldots \ldots \ldots \ldots \ldots \ldots 20 \ldots \ldots$

5.6. Energy-Bandwidth Plots Derived from $R_{0} \ldots \ldots \ldots \ldots \ldots \ldots \ldots \ldots \ldots$

References ........................................ 209

Chapter 6. Transmitters $\ldots \ldots \ldots \ldots \ldots \ldots \ldots \ldots \ldots \ldots \ldots \ldots \ldots, 211$

6.1. Read Only Memory Implementations $\ldots \ldots \ldots \ldots \ldots \ldots \ldots \ldots \ldots \ldots \ldots \ldots \ldots$

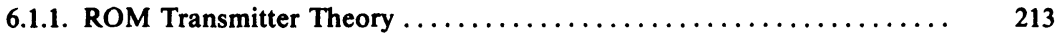

6.1.2. Practical Considerations for ROM Transmitters .............. 216

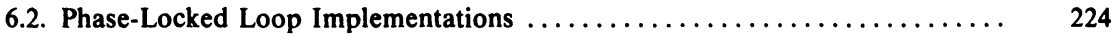

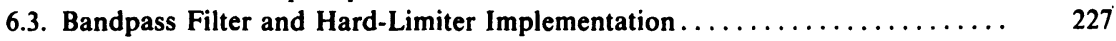

6.4. Serial and Parallel MSK Transmitters $\ldots \ldots \ldots \ldots \ldots \ldots \ldots \ldots \ldots \ldots \ldots \ldots \ldots \ldots$

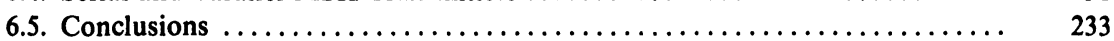

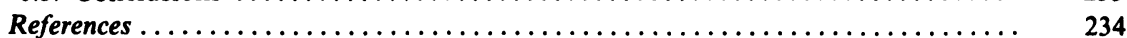

Chapter 7. Receivers $\ldots \ldots \ldots \ldots \ldots \ldots \ldots \ldots \ldots \ldots \ldots \ldots \ldots \ldots \ldots, 237$

7.1. Optimum Coherent and Noncoherent Receivers for CPFSK .......... 237

7.1.1. Optimum ML Coherent Receiver for CPFSK ................ 238

7.1.2. Optimal ML Noncoherent Receiver for CPFSK ............. 242

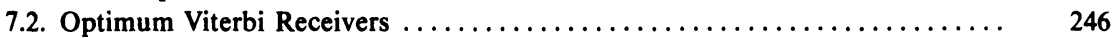

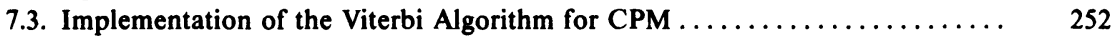

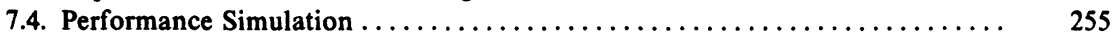

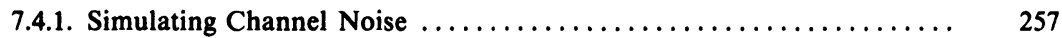

7.4.2. Realization of the Viterbi Algorithm and Numerical Results ........ 259

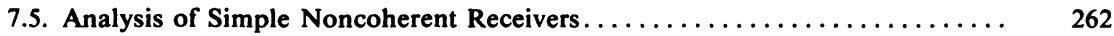

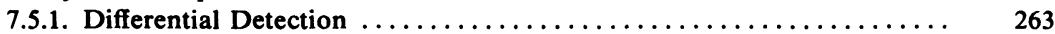

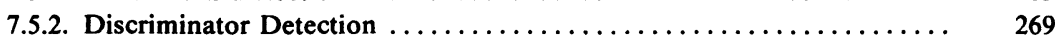

7.5.3. Numerical Results $\ldots \ldots \ldots \ldots \ldots \ldots \ldots \ldots \ldots \ldots \ldots \ldots \ldots \ldots \ldots \ldots . \ldots \ldots \ldots \ldots$

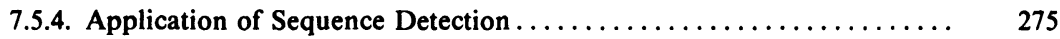

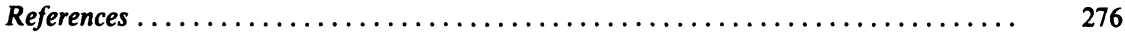

Chapter 8. Simplified Receivers .......................... 279

8.1. Reduced Complexity Viterbi Detectors ...................... 279

8.1.1. The Complexity Reduction Concept .................... 280

8.1.2. The Performance Measure ......................... 282

8.1.3. Properties of the Minimum Euclidean Distance for Mismatched Signal

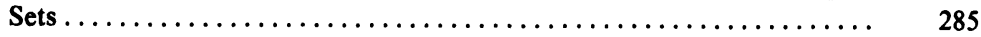

8.1.4. Optimizing the Reduced-Complexity Receiver .............. 287

8.1.5. Numerical Results ............................... 289

8.1.6. Summary and Tradeoff between Complexity Reduction and Performance 294

8.2. MSK-Type Receivers $\ldots \ldots \ldots \ldots \ldots \ldots \ldots \ldots \ldots \ldots \ldots \ldots \ldots \ldots \ldots \ldots . \ldots \ldots$

8.2.1. Receiver Principle and Performance ................... 296

8.2.2. The Optimum Receiver Filter ...................... 300

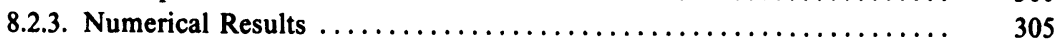

8.2.4. Discussion and Comparisons ..................... 309 
8.3. Serial MSK-Type Receivers ............................... 315

8.3.1. The Concept of Serial Detection ....................... 315

8.3.2. Error Probability Analysis $\ldots \ldots \ldots \ldots \ldots \ldots \ldots \ldots \ldots \ldots \ldots, \quad 317$

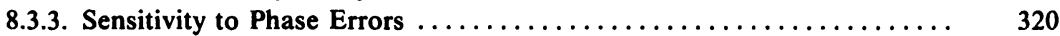

8.3.4. Sensitivity to Timing Errors $\ldots \ldots \ldots \ldots \ldots \ldots \ldots \ldots \ldots \ldots \ldots \ldots, 325$

8.3.5. Summary $\ldots \ldots \ldots \ldots \ldots \ldots \ldots \ldots \ldots \ldots \ldots \ldots \ldots \ldots \ldots \ldots \ldots \ldots, 328$

8.4. Average Matched Filter Receivers $\ldots \ldots \ldots \ldots \ldots \ldots \ldots \ldots \ldots \ldots \ldots \ldots, 329$

8.4.1. AMF Receiver Principles and Structure .................. 330

8.4.2. Error Performance of AMF Receivers ................... 333

8.4.3. Properties of the Distance and the Uniformly Weighted AMF ...... 341

8.4.4. Numerical Results ................................. 346

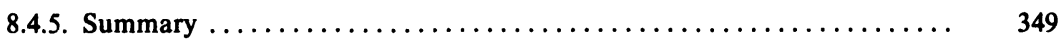

8.5. Limited Trellis Search Receivers ........................... 352

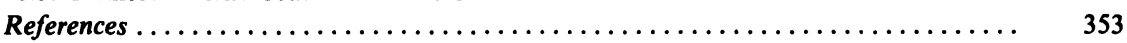

Chapter 9. Synchronization $\ldots \ldots \ldots \ldots \ldots \ldots \ldots \ldots \ldots \ldots \ldots \ldots \ldots \ldots \ldots$

9.1. Synchronization with a Specified Structure .................... 357

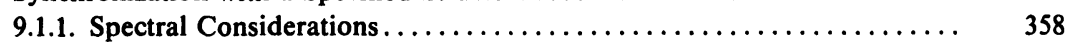

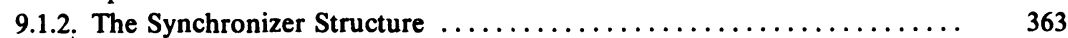

9.1.3. Discrete Component CNRs .......................... 364

9.1.4. Error Performance ............................. 367

9.1.5. Multi- $h$ Full Response Synchronization ................... 374

9.2. ML Estimation of Data, Phase, and Timing .................. 379

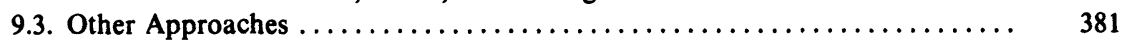

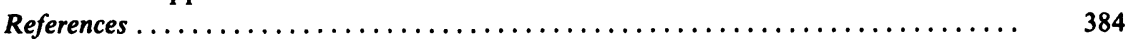

Chapter 10. Partially Coherent Receivers................... 387

10.1. Notions of Phase Coherence ............................ 387

10.2. Partially Coherent Detection; Equivalent Distance ................. 388

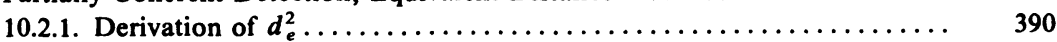

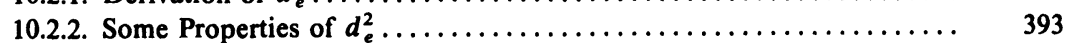

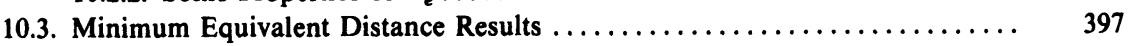

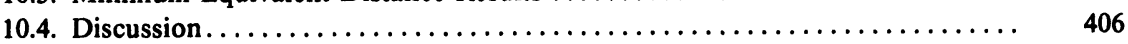

References $\ldots \ldots \ldots \ldots \ldots \ldots \ldots \ldots \ldots \ldots \ldots \ldots \ldots \ldots \ldots \ldots \ldots \ldots \ldots \ldots \ldots \ldots, 411$

Chapter 11. Phase Modulation and Convolutional Codes ......... 413

11.1. PSK Set Partition Codes $\ldots \ldots \ldots \ldots \ldots \ldots \ldots \ldots \ldots \ldots \ldots \ldots \ldots \ldots, 413$

11.2. Coded Binary and Quaternary CPFSK $\ldots \ldots \ldots \ldots \ldots \ldots \ldots \ldots \ldots \ldots, 417$

11.2.1. System Description ............................ 417

11.2.2. Error Probability and Minimum Euclidean Distance............ 419

11.2.3. Minimum Euclidean Distance: Properties and Calculation ........ 420

11.2.4. Symmetries for the Combination of Convolutional Encoder and Map-

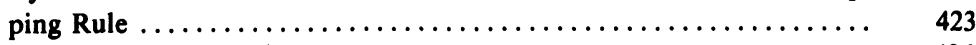

11.2.5. Numerical Results .............................. 426

11.2.6. Energy-Bandwidth Tradeoff $\ldots \ldots \ldots \ldots \ldots \ldots \ldots \ldots \ldots \ldots \ldots .437$ 


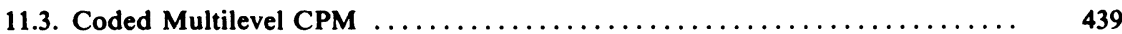

11.3.1. System Description .............................. 439

11.3.2. Bounds on the Free Euclidean Distance .................. 440

11.3.3. Code Search and Tables of Good Codes ................... 443

11.3.4. Energy and Bandwidth Efficiency of Coded CPM ............. 446

11.3.5. Conclusions .................................. 450

11.4. Simulations of Coded CPFSK Schemes $\ldots \ldots \ldots \ldots \ldots \ldots \ldots \ldots \ldots \ldots, 454$

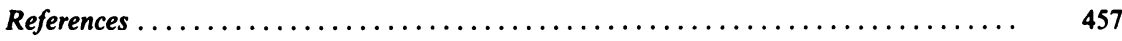

Appendix A. Minimum Distance Calculation Algorithms ........ 461

A.1. Limited Sequential Tree Search ......................... 462

A.2. The Viterbi Algorithm in a Superstate Trellis $\ldots \ldots \ldots \ldots \ldots \ldots \ldots \ldots, 465$

Appendix B. Specification of the Markov Distance Chain and Calculation of $\boldsymbol{R}_{0} \ldots \ldots \ldots \ldots \ldots \ldots \ldots, 469$

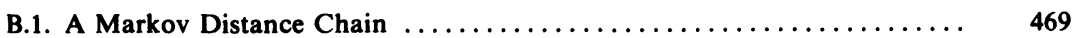

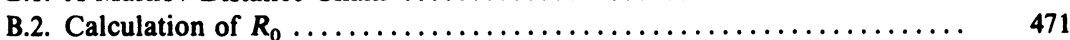

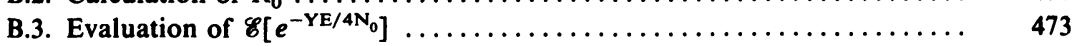

Appendix C. Simulation Techniques .................... 475

C.1. Statistical Analysis of Error Events ...................... 475

C.2. The Signal Space Method of Simulation $\ldots \ldots \ldots \ldots \ldots \ldots \ldots \ldots \ldots .480$

Appendix D. Tables of Optimum Codes .................... 483

D.1. Optimal Codes for Binary and Quaternary CPFSK ............. 483

D.2. Optimal Codes for 8-, 16- and 32-Level CPFSK $\ldots \ldots \ldots \ldots \ldots \ldots \ldots, 487$

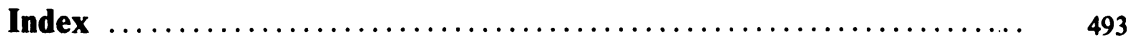

